\title{
The joint distribution of $Q$-additive functions on polynomials over finite fields
}

\author{
par Michael DRMOTA et Georg GUTENBRUNNER
}

RÉSUMÉ. Soient $K$ un corps fini et $Q \in K[T]$ un polynôme de degré au moins égal à 1 . Une fonction $f$ sur $K[T]$ est dite (complètement) $Q$-additive si $f(A+B Q)=f(A)+f(B)$ pour tous $A, B \in K[T]$ tels que $\operatorname{deg}(A)<\operatorname{deg}(Q)$. Nous montrons que les vecteurs $\left(f_{1}(A), \ldots, f_{d}(A)\right)$ sont asymptotiquement équirépartis dans l'ensemble image $\left\{\left(f_{1}(A), \ldots, f_{d}(A)\right): A \in K[T]\right\}$ si les $Q_{j}$ sont premiers entre eux deux à deux et si les $f_{j}: K[T] \rightarrow K[T]$ sont $Q_{j}$-additives. En outre, nous établissons que les vecteurs $\left(g_{1}(A), g_{2}(A)\right)$ sont asymptotiquement indépendants et gaussiens si $g_{1}, g_{2}: K[T] \rightarrow \mathbb{R}$ sont $Q_{1}$ - resp. $Q_{2}$-additives.

Abstract. Let $K$ be a finite field and $Q \in K[T]$ a polynomial of positive degree. A function $f$ on $K[T]$ is called (completely) $Q$-additive if $f(A+B Q)=f(A)+f(B)$, where $A, B \in K[T]$ and $\operatorname{deg}(A)<\operatorname{deg}(Q)$. We prove that the values $\left(f_{1}(A), \ldots, f_{d}(A)\right)$ are asymptotically equidistributed on the (finite) image set $\left\{\left(f_{1}(A), \ldots, f_{d}(A)\right): A \in K[T]\right\}$ if $Q_{j}$ are pairwise coprime and $f_{j}: K[T] \rightarrow K[T]$ are $Q_{j}$-additive. Furthermore, it is shown that $\left(g_{1}(A), g_{2}(A)\right)$ are asymptotically independent and Gaussian if $g_{1}, g_{2}: K[T] \rightarrow \mathbb{R}$ are $Q_{1}$ - resp. $Q_{2}$-additive.

\section{Introduction}

Let $g>1$ be a given integer. A function $f: \mathbb{N} \rightarrow \mathbb{R}$ is called (completely) $g$-additive if

$$
f(a+b g)=f(a)+f(b)
$$

for $a, b \in \mathbb{N}$ and $0 \leq a<g$. In particular, if $n \in \mathbb{N}$ is given in its $g$-ary expansion

This research was supported by the Austrian Science Foundation FWF, grant S8302-MAT. 


$$
n=\sum_{j \geq 0} \varepsilon_{g, j}(n) g^{j}
$$

then

$$
f(n)=\sum_{j \geq 0} f\left(\varepsilon_{g, j}(n)\right) .
$$

$g$-additive functions have been extensively discussed in the literature, in particular their asymptotic distribution, see $[1,3,4,5,6,7,8,9,11,12,14$, 15]. We cite three of these results (in a slightly modified form). We want to emphasise that Theorems $\mathrm{A}$ and $\mathrm{C}$ also say that different $g$-ary expansions are (asymtotically) independent if the bases are coprime.

Theorem A. (Kim [13]) Suppose that $g_{1}, \ldots, g_{d} \geq 2$ are pairwise coprime integers, $m_{1}, \ldots, m_{d}$ positive integers, and let $f_{j}, 1 \leq j \leq d$, be completely $g_{j}$-additive functions. Set

$$
H:=\left\{\left(f_{1}(n) \bmod m_{1}, \ldots, f_{d}(n) \bmod m_{d}\right): n \geq 0\right\} .
$$

Then $H$ is a subgroup of $\mathbb{Z}_{m_{1}} \times \cdots \times \mathbb{Z}_{m_{d}}$ and for every $\left(a_{1}, \ldots, a_{d}\right) \in H$ we have

$$
\frac{1}{N} \#\left\{\begin{array}{ccc}
f_{1}(n) \bmod m_{1}=a_{1}, \\
n<N: \begin{array}{c}
\vdots \\
f_{d}(n) \bmod m_{d}=
\end{array}
\end{array}\right\}=\frac{1}{|H|}+O\left(N^{-\delta}\right),
$$

where $\delta=1 /\left(120 d^{2} \bar{g}^{3} \bar{m}^{2}\right)$ with

$$
\bar{g}=\max _{1 \leq j \leq d} g_{j} \quad \text { and } \quad \bar{m}=\max _{1 \leq j \leq d} m_{j}
$$

and the $O$-constant depends only on $d$ and $g_{1}, \ldots, g_{d}$.

Remark. In [13] the set $H$ is explicitly determined. Set $F_{j}=f_{j}(1)$ and $d_{j}=\operatorname{gcd}\left\{m_{j},\left(q_{j}-1\right) F_{j}, f_{j}(r)-r F_{j}\left(2 \leq j \leq q_{j}-1\right)\right\}$. Then $\left(a_{1}, \ldots, a_{d}\right) \in H$ if and only if the system of congruences $F_{j} n \equiv a_{j} \bmod d_{j}, 1 \leq j \leq d$, has a solution.

Theorem B. (Bassily-Katai [1]) Let $f$ be a completely g-additive function and let $P(x)$ be a polynomial of degree $r$ with non-negative integer coefficients. Then, as $N \rightarrow \infty$,

$$
\frac{1}{N} \#\left\{n<N: \frac{f(P(n))-r \mu_{f} \log _{g} N}{\sqrt{r \sigma_{f}^{2} \log _{g} N}}<x\right\} \rightarrow \Phi(x)
$$

and

$$
\frac{1}{\pi(N)} \#\left\{p<N: p \text { prime }, \frac{f(P(p))-r \mu_{f} \log _{g} N}{\sqrt{r \sigma_{f}^{2} \log _{g} N}}<x\right\} \rightarrow \Phi(x),
$$


where

$$
\mu_{f}=\frac{1}{g} \sum_{r=0}^{g-1} f(r) \quad \text { and } \quad \sigma_{f}^{2}=\frac{1}{g} \sum_{r=0}^{g-1} f(r)^{2}-\mu_{f}^{2} \text {, }
$$

and

$$
\Phi(x)=\frac{1}{\sqrt{2 \pi}} \int_{-\infty}^{x} e^{-t^{2} / 2} d t
$$

Remark. The result of [1] is more general. It also provides asymptotic normality if $f$ is not strictly $g$-additive but the variance grows sufficiently fast.

Theorem C. (Drmota [6]) Suppose that $g_{1} \geq 2$ and $g_{2} \geq 2$ are coprime integers and that $f_{1}$ and $f_{2}$ are completely $g_{1}$-resp. $g_{2}$-additive functions. Then, as $N \rightarrow \infty$,

$$
\begin{aligned}
& \frac{1}{N} \#\left\{n<N: \frac{f_{1}(n)-\mu_{f_{1}} \log _{g_{1}} N}{\sqrt{\sigma_{f_{1}}^{2} \log _{g_{1}} N}} \leq x_{1}, \frac{f_{2}(n)-\mu_{f_{2}} \log _{g_{2}} N}{\sqrt{\sigma_{f_{2}}^{2} \log _{g_{2}} N}} \leq x_{2}\right\} \\
& \rightarrow \Phi\left(x_{1}\right) \Phi\left(x_{2}\right) \text {. }
\end{aligned}
$$

Remark. Here it is also possible to provide general versions (see Steiner [17]) but - up to now - it was not possible to prove a similar property for three or more bases $g_{j}$.

The purpose of this paper is to generalize these kinds of result to polynomials over finite fields.

Let $\mathbb{F}_{q}$ be a finite field of characteristic $p$ (that is, $q=\left|\mathbb{F}_{q}\right|$ is a power of $p$ ) and let $\mathbb{F}_{q}[T]$ denotes the ring of polynomials over $\mathbb{F}_{q}$. The set of polynomials in $\mathbb{F}_{q}$ of degree $<k$ will be denoted by $P_{k}=\left\{A \in \mathbb{F}_{q}[T]\right.$ : $\operatorname{deg} A<k\}$. Fix some polynomial $Q \in \mathbb{F}_{q}[T]$ of positive degree. A function $f: \mathbb{F}_{q}[T] \rightarrow G$ (where $G$ is any abelian group) is called (completely) $Q$-additive if $f(A+B Q)=f(A)+f(B)$, where $A, B \in \mathbb{F}_{q}[T]$ and $\operatorname{deg}(A)<\operatorname{deg}(Q)$. More precisely, if a polynomial $A \in \mathbb{F}_{q}[T]$ is represented in its $Q$-ary digital expansion

$$
A=\sum_{j \geq 0} D_{Q, j}(A) Q^{j}
$$

where $D_{Q, j}(A) \in P_{k}$ are the digits, that is, polynomials of degree smaller than $k=\operatorname{deg} Q$, then

$$
f(A)=\sum_{j \geq 0} f\left(D_{Q, j}(A)\right)
$$


For example, the sum-of-digits function $s_{Q}: \mathbb{F}_{q}[T] \rightarrow \mathbb{F}_{q}[T]$ is defined by

$$
s_{Q}(A)=\sum_{j \geq 0} D_{Q, j}(A) .
$$

Note that the image set of a $Q$-additive function is always finite and that (in contrast to the integer case) the sum-of-digits function satisfies $s_{Q}(A+B)=$ $s_{Q}(A)+s_{Q}(B)$.

\section{Results}

The first theorem is a direct generalization of Theorem A.

Theorem 2.1. Let $Q_{1}, Q_{2}, \ldots, Q_{d}$ and $M_{1}, M_{2}, \ldots, M_{d}$ be non-zero polynomials in $\mathbb{F}_{q}[T]$ with $\operatorname{deg} Q_{i}=k_{i}, \operatorname{deg} M_{i}=m_{i}$ and $\left(Q_{i}, Q_{j}\right)=1$ for $i \neq j$. Furthermore let $f_{i}: \mathbb{F}_{q}[T] \rightarrow \mathbb{F}_{q}[T]$ be $Q_{i}$-additive functions $(1 \leq i \leq d)$. Set

$$
H:=\left\{\left(f_{1}(A) \bmod M_{1}, \ldots, f_{d}(A) \bmod M_{d}\right): A \in \mathbb{F}_{q}[T]\right\} .
$$

Then $H$ is a subgroup of $P_{m_{1}} \times \cdots \times P_{m_{d}}$ and for every $\left(R_{1}, \ldots, R_{d}\right) \in H$ we have

$\lim _{l \rightarrow \infty} \frac{1}{q^{l}} \#\left\{A \in P_{l}: f_{1}(A) \bmod M_{1}=R_{1}, \ldots, f_{d}(A) \bmod M_{d}=R_{d}\right\}=\frac{1}{|H|}$.

Since the image sets of $f_{i}$ are finite we can choose the degrees $m_{i}$ of $M_{i}$ sufficiently large and obtain

$$
\lim _{l \rightarrow \infty} \frac{1}{q^{l}} \#\left\{A \in P_{l}: f_{1}(A)=R_{1}, \ldots, f_{d}(A)=R_{d}\right\}=\frac{1}{\left|H^{\prime}\right|},
$$

where

$$
H^{\prime}:=\left\{\left(f_{1}(A), \ldots, f_{d}(A)\right): A \in \mathbb{F}_{q}[T]\right\} .
$$

In particular this theorem says that if there is $A \in \mathbb{F}_{q}[T]$ with $f_{i}(A)=R_{i}$ $(1 \leq i \leq d)$ then there are infinitely many $A \in \mathbb{F}_{q}[T]$ with that property.

The next theorem is a generalization of Theorem B.

Theorem 2.2. Let $Q \in \mathbb{F}_{q}[T], k=\operatorname{deg} Q \geq 1$ be a given polynomial, $g: \mathbb{F}_{q}[T] \rightarrow \mathbb{R}$ be a $Q$-additive function, and set

$$
\mu_{g}:=\frac{1}{q^{k}} \sum_{A \in P_{k}} g(A), \quad \sigma_{g}^{2}:=\frac{1}{q^{k}} \sum_{A \in P_{k}} g(A)^{2}-\mu_{g}^{2} .
$$

Let $P(T) \in \mathbb{F}_{q}[T]$ with $r=\operatorname{deg} P$ and suppose that $\sigma_{g}^{2}>0$. Then, as $n \rightarrow \infty$,

$$
\frac{1}{q^{n}} \#\left\{A \in P_{n}: \frac{g(P(A))-\frac{n r}{k} \mu_{g}}{\sqrt{\frac{n r}{k} \sigma_{g}^{2}}} \leq x\right\} \rightarrow \Phi(x)
$$


and

$$
\frac{1}{\left|I_{n}\right|} \#\left\{A \in I_{n}: \frac{g(P(A))-\frac{n r}{k} \mu_{g}}{\sqrt{\frac{n r}{k} \sigma_{g}^{2}}} \leq x\right\} \rightarrow \Phi(x),
$$

where $I_{n}$ denotes the set of monic irreducible polynomials of degree $<n$.

Finally we present a generalization of Theorem C.

Theorem 2.3. Suppose that $Q_{1} \in \mathbb{F}_{q}[T]$ and $Q_{2} \in \mathbb{F}_{q}[T]$ are coprime polynomials of degrees $k_{1} \geq 1$ resp. $k_{2} \geq 1$ such that at least one of the derivatives $Q_{1}^{\prime}, Q_{2}^{\prime}$ is non-zero. Further suppose that $g_{1}: \mathbb{F}_{q}[T] \rightarrow \mathbb{R}$ and $g_{2}: \mathbb{F}_{q}[T] \rightarrow \mathbb{R}$ are completely $Q_{1}$ - resp. $Q_{2}$-additive functions.

Then, as $n \rightarrow \infty$,

$$
\begin{aligned}
\frac{1}{q^{n}} \# & \left\{A \in P_{n}: \frac{g_{1}(A)-\frac{n}{k_{1}} \mu_{g_{1}}}{\sqrt{\frac{n}{k_{1}} \sigma_{g_{1}}^{2}}} \leq x_{1}, \frac{g_{2}(A)-\frac{n}{k_{2}} \mu_{g_{2}}}{\sqrt{\frac{n}{k_{2}} \sigma_{g_{2}}^{2}}} \leq x_{2}\right\} \\
& \rightarrow \Phi\left(x_{1}\right) \Phi\left(x_{2}\right) .
\end{aligned}
$$

Furthermore, Theorems 2.1 and 2.3 say that $Q$-ary digital expansions are (asymptotically) independent if the base polynomials are pairwise coprime.

\section{Proof of Theorem 2.1}

Throughout the paper we will use the additive character $E$ defined by

$$
E(A):=e^{2 \pi i \operatorname{tr}(\operatorname{Res}(A)) / p},
$$

that is defined for all formal Laurent series

$$
A=\sum_{j \geq-k} a_{j} T^{-j}
$$

with $k \in \mathbb{Z}$ and $a_{j} \in \mathbb{F}_{q}$. The residue $\operatorname{Res}(A)$ is given by $\operatorname{Res}(A)=a_{1}$ and tr is the usual trace $\operatorname{tr}: \mathbb{F}_{q} \rightarrow \mathbb{F}_{p}$.

Let $Q_{1}, Q_{2}, \ldots, Q_{d}$ and $M_{1}, M_{2}, \ldots, M_{d}$ be non-zero polynomials in $\mathbb{F}_{q}[T]$ with $\operatorname{deg} Q_{i}=k_{i}, \operatorname{deg} M_{i}=m_{i}$ and $\left(Q_{i}, Q_{j}\right)=1$ for $i \neq j$. Furthermore let $f_{i}$ be completely $Q_{i}$-additive functions. For every tuple $R=\left(R_{1}, \ldots, R_{d}\right) \in$ $P_{m_{1}} \times \cdots \times P_{m_{d}}$ set

$$
g_{R_{i}}(A):=E\left(\frac{R_{i}}{M_{i}} f_{i}(A)\right)
$$

and 


$$
g_{R}(A):=\prod_{i=1}^{d} g_{R_{i}}(A)=E\left(\sum_{i=1}^{d} \frac{R_{i}}{M_{i}} f_{i}(A)\right) .
$$

Proposition 3.1. Let $Q_{1}, Q_{2}, \ldots, Q_{d}, M_{1}, M_{2}, \ldots, M_{d}$, and $R=\left(R_{1}, \ldots\right.$, $\left.R_{d}\right)$ be as above. Then we either have

$$
g_{R}(A)=1 \quad \text { for all } A \in \mathbb{F}_{q}[T]
$$

or

$$
\lim _{l \rightarrow \infty} \frac{1}{q^{l}} \sum_{A \in P_{l}} g_{R}(A)=0
$$

We will first prove Proposition 3.1 (following the lines of Kim [13]). Theorem 2.1 is then an easy corollary.

\subsection{Preliminaries.}

Lemma 3.1. Let $H \neq 0, H, G \in \mathbb{F}_{q}[T]$, and let $E$ be the character defined in (3.1), then:

$$
\sum_{\operatorname{deg} R<\operatorname{deg} H} E\left(\frac{G}{H} R\right)=\left\{\begin{array}{cl}
q^{\operatorname{deg} H} & \text { if } H \text { divides } G \\
0 & \text { otherwise. }
\end{array}\right.
$$

The next lemma is a version of the Weyl-van der Corput inequality.

Lemma 3.2. For each $A \in \mathbb{F}_{q}[T]$ let $u_{A}$ be a complex number, with $\left|u_{A}\right|=$ 1 , then

$$
\left|\frac{1}{q^{l}} \sum_{A \in P_{l}} u_{A}\right|^{2} \leq \frac{1}{q^{r}}+\frac{1}{q^{r}} \sum_{D \in P_{r} \backslash\{0\}}\left|\frac{1}{q^{l}} \sum_{A \in P_{l}} \overline{u_{A}} u_{A+D}\right| .
$$

Proof. Since $\left\langle P_{l},+\right\rangle$ is a group we have

$$
\begin{aligned}
q^{r} \sum_{A \in P_{l}} u_{A} & =\sum_{B \in P_{r}} \sum_{A \in P_{l}} u_{A-B} \\
& =\sum_{A \in P_{l}} 1\left(\sum_{B \in P_{r}} u_{A-B}\right) .
\end{aligned}
$$


Hence, using the Cauchy-Schwarz-inequality

$$
\begin{aligned}
q^{2 r}\left|\sum_{A \in P_{l}} u_{A}\right|^{2} & \leq \sum_{A \in P_{l}} 1^{2} \sum_{A \in P_{l}}\left|\sum_{B \in P_{r}} u_{A-B}\right|^{2} \\
& =q^{l} \sum_{A \in P_{l}} \sum_{B \in P_{r}} \sum_{C \in P_{r}} \bar{u}_{A-B} u_{A-C} \\
& =q^{l} \sum_{D \in P_{r}} \sum_{A \in P_{l}} \sum_{B \in P_{r}} \bar{u}_{A-B} u_{A-B+D} \\
& =q^{l} \sum_{D \in P_{r}} \sum_{B \in P_{r}} \sum_{A \in P_{l}} \bar{u}_{A-B} u_{A-B+D} \\
& =q^{l+r} \sum_{D \in P_{r}} \sum_{A \in P_{l}} \bar{u}_{A} u_{A+D} \\
& =q^{l+r} \sum_{A \in P_{l}}\left|u_{A}\right|^{2}+q^{l+r} \sum_{D \in P_{r} \backslash\{0\}} \sum_{A \in P_{l}} \bar{u}_{A} u_{A+D} .
\end{aligned}
$$

The desired result follows from $\left|u_{A}\right|=1$.

Lemma 3.3. Let $f$ be a completely $Q$-additive function, and $t \in \mathbb{N}, K, R \in$ $\mathbb{F}_{q}[T]$ with $\operatorname{deg} R, \operatorname{deg} K<\operatorname{deg} Q^{t}$. Then for all $N \in \mathbb{F}_{q}[T]$ satisfying $N \equiv R \bmod Q^{t}$ we have

$$
f(N+K)-f(N)=f(R+K)-f(R) .
$$

Proof. Due to the above conditions, $N=A \cdot Q^{t}+R$ for some $A \in \mathbb{F}_{q}[T]$. Since $f$ is completely $Q$-additive, and $\operatorname{deg}(R+K)<\operatorname{deg}\left(Q^{t}\right)$, we have

$$
\begin{aligned}
f(N+K)-f(N) & =f\left(A Q^{t}+R+K\right)-f\left(A Q^{t}+R\right) \\
& =f(A)+f(R+K)-(f(A)+f(R)) \\
& =f(R+K)-f(R) .
\end{aligned}
$$

3.2. Correlation Estimates. In this section we will first prove a correlation estimate (Lemma 3.4) which will be applied to prove a pre-version (Lemma 3.5) of Proposition 3.1.

Let $Q \in \mathbb{F}_{q}[T]$ of $\operatorname{deg} Q=k, M \in \mathbb{F}_{q}[T]$ of $\operatorname{deg} M=m$, and $f$ be a (completely) $Q$-additive function. Furthermore for $R \in P_{m}$ set $g(A):=$ $E\left(\frac{R}{M} f(A)\right)$.

Unless otherwise specified, $n$ and $l$ are arbitrary integers, and $D \in \mathbb{F}_{q}[T]$ arbitrary as well. We introduce the correlation functions

$$
\Phi_{n}(D)=\frac{1}{q^{n}} \sum_{A \in P_{n}} \overline{g(A)} g(A+D)
$$


and

$$
\Phi_{l, n}=\frac{1}{q^{l}} \sum_{A \in P_{l}}\left|\Phi_{n}(A)\right|^{2} .
$$

Lemma 3.4. Suppose that $\left|\Phi_{k}(R)\right|<1$. Then

$$
\begin{aligned}
\frac{1}{q^{l}} \sum_{H \in P_{l}}\left|\frac{1}{q^{n}} \sum_{A \in P_{n}} E\left(\frac{R}{M}(f(A+H)-f(A))\right)\right|^{2} \\
\ll \exp \left(-\min \{n, l\} \frac{1-\left|\Phi_{k}(R)\right|^{2}}{k q^{k}}\right) .
\end{aligned}
$$

Proof. We begin by establishing some recurrence relations for $\Phi_{n}$ and $\Phi_{l, n}$, namely

$$
\Phi_{k+n}(Q K+R)=\Phi_{k}(R) \Phi_{n}(K)
$$

for polynomials $K, R$ with $R \in P_{k}$. By using the relation $g(A Q+B)=$ $g(A) g(B)$ and splitting the sum defining $\Phi_{k+n}(Q K+R)$ according to the residue class of $A$ modulo $Q$ we obtain

$$
\begin{aligned}
q^{k+n} \Phi_{k+n}(Q K+R) & =\sum_{I \in P_{k}} \sum_{A \in P_{n}} \overline{g(A Q+I)} g(A Q+I+Q K+R) \\
& =\sum_{I \in P_{k}} \sum_{A \in P_{n}} \overline{g(A) g(I)} g(A+K) g(I+R) \\
& =\sum_{I \in P_{k}} \overline{g(I)} g(I+R) \sum_{A \in P_{n}} \overline{g(A)} g(A+K) \\
& =q^{k} \Phi_{k}(R) q^{n} \Phi_{n}(K) .
\end{aligned}
$$

This proves (3.8).

Next observe that

$$
\begin{aligned}
q^{k+l} \Phi_{k+l, k+n} & =\sum_{I \in P_{k}} \sum_{A \in P_{l}} \overline{\Phi_{k+n}(Q A+I)} \Phi_{k+n}(Q A+I) \\
& =\sum_{I \in P_{k}} \sum_{A \in P_{l}} \overline{\Phi_{k}(I) \Phi_{n}(A)} \Phi_{k}(I) \Phi_{n}(A) \\
& =\sum_{I \in P_{k}} \overline{\Phi_{k}(I)} \Phi_{k}(I) \sum_{A \in P_{l}} \overline{\Phi_{n}(A)} \Phi_{n}(A) \\
& =q^{k} \Phi_{k, k} q^{l} \Phi_{l, n} .
\end{aligned}
$$

Thus

$$
\Phi_{k+l, k+n}=\Phi_{k, k} \Phi_{l, n}
$$


and consequently

$$
\Phi_{i k+l, i k+n}=\left(\Phi_{k, k}\right)^{i} \Phi_{l, n} .
$$

Since $\left|\Phi_{l, n}\right| \leq 1$ we also get $\left|\Phi_{i k+l, i k+n}\right| \leq\left|\Phi_{k, k}\right|^{i}$.

Hence, if $n$ and $l$ are given then we can represent them as $n=i k+r, l=$ $i k+s$ with $i=\min ([n / k],[l / k])$ and $\min (r, s)<k$. By definition we have

$$
\Phi_{k, k}=\frac{1}{q^{k}} \sum_{A \in P_{k}}\left|\Phi_{k}(A)\right|^{2}
$$

with $\left|\Phi_{k}(A)\right| \leq 1$ for all $A$. Since $\left|\Phi_{k}(R)\right|<1$ we also have

$$
\Phi_{k, k} \leq 1-\frac{1-\left|\Phi_{k}(R)\right|^{2}}{q^{k}} \leq \exp \left(-\frac{1-\left|\Phi_{k}(R)\right|^{2}}{q^{k}}\right)<1
$$

and consequently

$$
\left|\Phi_{l, n}\right| \leq\left|\Phi_{k, k}\right|^{i} \ll \exp \left(-\min \{l, n\} \frac{1-\left|\Phi_{k}(R)\right|^{2}}{k q^{k}}\right) .
$$

Remark. We want to remark that $\left|\Phi_{k}(R)\right|=1$ is a rare event. In particular, we have

$$
\begin{aligned}
\forall R:\left|\Phi_{k}(R)\right|=1 & \Leftrightarrow \forall R \forall A \in P_{k}: \overline{g(A)} g(A+R) \text { is constant } \\
& \Leftrightarrow \forall R, \forall A, B \in P_{k}: \overline{g(A)} g(A+R)=\overline{g(B)} g(B+R) \\
& \Leftrightarrow \forall A, B \in P_{k}: g(A+B)=g(A) g(B) .
\end{aligned}
$$

Thus, there exists $R$ with $\left|\Phi_{k}(R)\right|<1$ if and only if there exist $A, B \in P_{k}$ with $g(A) g(B) \neq g(A+B)$.

Next we prove a pre-version of Proposition 3.1.

Lemma 3.5. Let $Q_{1}, Q_{2}, \ldots, Q_{d} \in \mathbb{F}_{q}[T]$ be pairwise coprime polynomials, $M_{1}, M_{2}, \ldots, M_{d} \in \mathbb{F}_{q}[T]$, and $R=\left(R_{1}, R_{2}, \ldots, R_{d}\right) \in P_{m_{1}} \times \cdots \times P_{m_{d}}$ such that $\left|\Phi_{k_{j}}\left(R_{j}\right)\right|<1$ for at least one $j=1, \ldots, d$. Then

$$
\lim _{l \rightarrow \infty} \frac{1}{q^{l}} \sum_{A \in P_{l}} g_{R}(A)=0
$$

where $g_{R}(A)=\prod_{j=1}^{d} g_{R_{j}}(A)$ with $g_{R_{j}}(A)=E\left(\frac{R_{j}}{M_{j}} f_{j}(A)\right)$. 
Proof. Set $B_{j}=Q_{j}^{t_{j}}$ and suppose that $b_{j}=t_{j} \operatorname{deg} Q_{j}$ satisfies that $r \leq b_{j} \leq$ $2 r$ with $r=\frac{l}{3 d}$. Given $S=\left(S_{1}, S_{2}, \ldots, S_{d}\right)$ and $B_{1}, B_{2}, \ldots, B_{d}$, we define $N_{S}:=\left\{A \in P_{l}: A \equiv S_{1} \bmod B_{1}, \ldots, A \equiv S_{d} \bmod B_{d}\right\}$. By the Chinese remainder theorem we have for $l \geq \sum_{j=1}^{d} b_{j}$

$$
\left|N_{S}\right|=\frac{q^{l}}{\prod_{j=1}^{d} q^{b_{j}}}=q^{l-\sum_{j=1}^{d} b_{j}}
$$

Furthermore set $\mathcal{S}:=P_{b_{1}} \times \cdots \times P_{b_{d}}$. By Lemma 3.3 we obtain for $D \in P_{r} \backslash\{0\}:$

$$
\begin{aligned}
\sum_{A \in P_{l}} \overline{g_{R}(A)} g_{R}(A+D) & =\sum_{S \in \mathcal{S}} \sum_{A \in N_{S}} \overline{g_{R}(A)} g_{R}(A+D) \\
& =\sum_{S \in \mathcal{S}} \sum_{A \in N_{S}} \prod_{j=1}^{d} \overline{g_{R_{j}}\left(S_{j}\right)} g_{R_{j}}\left(S_{j}+D\right) \\
& =\sum_{S \in \mathcal{S}} \prod_{j=1}^{d} \overline{g_{R_{j}}\left(S_{j}\right)} g_{R_{j}}\left(S_{j}+D\right) \sum_{A \in N_{S}} 1 \\
& =\prod_{j=1}^{d} \sum_{S_{j} \in P_{b_{j}}} \overline{g_{R_{j}}\left(S_{j}\right)} g_{R_{j}}\left(S_{j}+D\right) \frac{q^{l}}{\prod_{j=1}^{d} q^{b_{j}}} \\
& =q^{l} \prod_{j=1}^{d} \frac{1}{q^{b_{j}}} \sum_{S_{j} \in P_{b_{j}}} \overline{g_{R_{j}}\left(S_{j}\right)} g_{R_{j}}\left(S_{j}+D\right) .
\end{aligned}
$$

According to Lemma 3.2 we obtain for $r \leq l$

$$
\begin{aligned}
\left|\sum_{A \in P_{l}} g_{R}(A)\right|^{2} \leq & q^{2 l-r}+q^{l-r} \sum_{D \in P_{r} \backslash\{0\}}\left|\sum_{A \in P_{l}} \overline{g_{R}(A)} g_{R}(A+D)\right| \\
= & q^{2 l-r} \underbrace{\sum_{D \in P_{r} \backslash\{0\}}\left|\prod_{j=1}^{d} q^{-b_{j}} \sum_{S_{j} \in P_{b_{j}}} \overline{g_{R_{j}}\left(S_{j}\right)} g_{R_{j}}\left(S_{j}+D\right)\right|}_{\Sigma_{1}} \\
& +O\left(q^{2 l-r}\right) .
\end{aligned}
$$


Hölder's inequality gives

$$
\begin{aligned}
\Sigma_{1} & \leq q^{r /(d+1)} \prod_{j=1}^{d}\left(\sum_{D \in P_{r} \backslash\{0\}}\left|q^{-b_{j}} \sum_{S_{j} \in P_{b_{j}}} \overline{g_{R_{j}}\left(S_{j}\right)} g_{R_{j}}\left(S_{j}+D\right)\right|^{d+1}\right)^{1 /(d+1)} \\
& \leq q^{r} \prod_{j=1}^{d}\left(q^{-r} \sum_{D \in P_{r} \backslash\{0\}}\left|q^{-b_{j}} \sum_{S_{j} \in P_{b_{j}}} \overline{g_{R_{j}}\left(S_{j}\right)} g_{R_{j}}\left(S_{j}+D\right)\right|^{2}\right)^{1 /(d+1)} .
\end{aligned}
$$

For some $j$ we have $\left|\Phi_{k_{j}}\left(R_{j}\right)\right|<1$, so that Lemma 3.4 is applicable and thus

$$
q^{-r} \sum_{D \in P_{r} \backslash\{0\}}\left|q^{-b_{j}} \sum_{S_{j} \in P_{b_{j}}} \overline{g_{R_{j}}\left(S_{j}\right)} g_{R_{j}}\left(S_{j}+D\right)\right|^{2} \rightarrow 0
$$

as $r=l /(3 d) \rightarrow \infty$. For all other $j$ we trivially estimate by $\leq 1$ and obtain

$$
\frac{1}{q^{l}}\left|\sum_{A \in P_{l}} g_{R}(A)\right| \rightarrow 0
$$

as $l \rightarrow \infty$.

3.3. Proof of Proposition 3.1. As above we set $g_{R}(A)=\prod_{j=1}^{d} g_{R_{j}}(A)=$ $E\left(\sum_{j=1}^{d} \frac{R_{j}}{M_{j}} f_{j}(A)\right)$. We split up the proof into several cases.

Case 1: There exist $j$ and $A, B \in P_{k_{j}}$ with $g_{R_{j}}(A) g_{R_{j}}(B) \neq g_{R_{j}}(A+B)$.

This case is covered by Lemma 3.5 (compare with the remark following Lemma 3.4).

Case 2: For all $j$ and for all $A, B \in P_{k_{j}}$ we have $g_{R_{j}}(A) g_{R_{j}}(B)=$ $g_{R_{j}}(A+B)$.

In this case we also have (due to the additivity property) $g_{R_{j}}(A) g_{R_{j}}(B)=$ $g_{R_{j}}(A+B)$ for all $A, B \in \mathbb{F}_{q}[T]$ and consequently $g(A) g(B)=g(A+B)$ for all $A, B \in \mathbb{F}_{q}[T]$.

Case 2.1: In addition we have $g(A)=1$ for all $A \in \mathbb{F}_{q}[T]$.

This case is the first alternative in Proposition 3.1. 
Case 2.2: In addition there exists $A \in \mathbb{F}_{q}[T]$ with $g(A) \neq 1$.

For simplicity we assume that $q$ is a prime number. Thus, if $A=$ $\sum_{i \geq 0} a_{i} T^{i}$ then we have $g(A)=\prod_{i \geq 0} g\left(T^{i}\right)^{a_{i}}$. Consequently there exists $i \geq 0$ with $g\left(T^{i}\right) \neq 1$. Furthermore

$$
\sum_{a=0}^{q-1} g\left(T^{j}\right)^{a}= \begin{cases}q & \text { if } g\left(T^{j}\right)=1 \\ 0 & \text { if } g\left(T^{j}\right) \neq 1\end{cases}
$$

Hence, if $l>i$ we surely have

$$
\begin{aligned}
\sum_{A \in P_{l}} g(A) & =\sum_{a_{0}=0}^{q-1} \sum_{a_{1}=0}^{q-1} \cdots \sum_{a_{l-1}=0}^{q-1} g\left(T^{0}\right)^{a_{0}} g\left(T^{1}\right)^{a_{1}} \cdots g\left(T^{l-1}\right)^{a_{l-1}} \\
& =\left(\sum_{a_{0}=0}^{q-1} g\left(T^{0}\right)^{a_{0}}\right) \cdots\left(\sum_{a_{l-1}=0}^{q-1} g\left(T^{l-1}\right)^{a_{l-1}}\right) \\
& =0
\end{aligned}
$$

If $q$ is a prime power the can argue in a similar way. This completes the proof of Proposition 3.1.

3.4. Completion of the Proof of Theorem 2.1. We define two (additive) groups

$$
G:=\left\{R=\left(R_{1}, R_{2}, \ldots, R_{d}\right) \in P_{m_{1}} \times \cdots \times P_{m_{d}}: \forall A \in \mathbb{F}_{q}[T] g_{R}(A)=1\right\}
$$

and

$$
H_{0}:=\left\{S \in P_{m_{1}} \times \cdots \times P_{m_{d}}: \forall R \in G E\left(\sum_{i=1}^{d}-\frac{S_{i} R_{i}}{M_{i}}\right)=1\right\}
$$

Furthermore, set

$$
F(S):=\frac{1}{|G|} \sum_{R \in G} E\left(\sum_{i=1}^{d}-\frac{S_{i} R_{i}}{M_{i}}\right) .
$$


Now, by applying Proposition 3.1 we directly get

$$
\begin{aligned}
& \frac{1}{q^{l}} \#\left\{A \in P_{l}: f_{1}(A) \equiv S_{1} \bmod M_{1}, \ldots, f_{d}(A) \equiv S_{d} \bmod M_{d}\right\}= \\
& =\frac{1}{q^{l}} \sum_{A \in P_{l}} \frac{1}{q^{\sum_{j=1}^{d} m_{j}}} \sum_{R \in P_{m_{1}} \times \cdots \times P_{m_{d}}} E\left(\sum_{j=1}^{d} \frac{R_{j}}{M_{j}}\left(f_{j}(A)-S_{j}\right)\right) \\
& =\frac{1}{q^{\sum_{j=1}^{d} m_{j}}} \sum_{R \in P_{m_{1} \times \cdots \times P_{m_{d}}}}\left[E\left(\sum_{j=1}^{d}-\frac{S_{j} R_{j}}{M_{j}}\right) \cdot \frac{1}{q^{l}} \sum_{A \in P_{l}} g_{R}(A)\right] \\
& =\frac{1}{q^{\sum_{j=1}^{d} m_{j}}} \sum_{R \in G} E\left(\sum_{j=1}^{d}-\frac{S_{j} R_{j}}{M_{j}}\right)+o(1) \\
& =\frac{|G|}{q^{\sum_{j=1}^{d} m_{j}}} F(S)+o(1) .
\end{aligned}
$$

More precisely the coefficient $F(S)$ characterizes $H_{0}$.

\section{Lemma 3.6. We have}

(1) $F(S)=1$ for $S \in H_{0}$

(2) $F(S)=0$ for $S \notin H_{0}$.

Furthermore $|G| \cdot\left|H_{0}\right|=\left|P_{m_{1}} \times \cdots \times P_{m_{d}}\right|=q^{m_{1}+\cdots+m_{d}}$.

Proof. It is clear that $F(S)=1$ if $S \in H_{0}$.

Now suppose that $S \notin H_{0}$. Then there exists $R^{0}=\left(R_{1}^{0}, R_{2}^{0}, \ldots, R_{d}^{0}\right) \in G$ with $E\left(\sum_{i=1}^{d}-\frac{S_{i} R_{i}^{0}}{M_{i}}\right) \neq 1$. Since

$$
\begin{aligned}
\sum_{R \in G} E\left(\sum_{i=1}^{d}-\frac{S_{i} R_{i}}{M_{i}}\right) & =\sum_{R \in G} E\left(\sum_{i=1}^{d}-\frac{S_{i}\left(R_{i}+R_{i}^{0}\right)}{M_{i}}\right) \\
& =E\left(\sum_{i=1}^{d}-\frac{S_{i} R_{i}^{0}}{M_{i}}\right) \sum_{R \in G} E\left(\sum_{i=1}^{d}-\frac{S_{i} R_{i}}{M_{i}}\right)
\end{aligned}
$$

it follows that $F(S)=0$.

Finally, by summing up over all $S \in P_{m_{1}} \times \cdots \times P_{m_{d}}$ it follows that $|G| \cdot\left|H_{0}\right|=\left|P_{m_{1}} \times \cdots \times P_{m_{d}}\right|$.

In fact we have now shown that (as $l \rightarrow \infty)$

$\frac{1}{q^{l}} \#\left\{A \in P_{l}: f_{1}(A) \equiv S_{1} \bmod M_{1}, \ldots, f_{d}(A) \equiv S_{d} \bmod M_{d}\right\}=\frac{1}{\left|H_{0}\right|}+o(1)$ if $S=\left(S_{1}, \ldots, S_{d}\right) \in H_{0}$ and $($ as $l \rightarrow \infty)$

$$
\frac{1}{q^{l}} \#\left\{A \in P_{l}: f_{1}(A) \equiv S_{1} \bmod M_{1}, \ldots, f_{d}(A) \equiv S_{d} \bmod M_{d}\right\}=o(1)
$$


if $S=\left(S_{1}, \ldots, S_{d}\right) \notin H_{0}$. The final step of the proof of Theorem 2.1 is to show that

$$
H=\left\{\left(f_{1}(A) \bmod M_{1}, \ldots, f_{d}(A) \bmod M_{d}\right): A \in \mathbb{F}_{q}[T]\right\}=H_{0} .
$$

In fact, if $S \in H_{0}$ then we trivially have $S \in H$.

Conversely, if $S \in H$ then there exists $A \in \mathbb{F}_{q}[T]$ with $f_{1}(A) \equiv S_{1}$ $\bmod M_{1}, \ldots, f_{d}(A) \equiv S_{d} \bmod M_{d}$. In particular, it follows that

$$
g_{R}(A)=E\left(\sum_{j=1}^{d} \frac{R_{j}}{M_{j}} f_{j}(A)\right)=E\left(\sum_{j=1}^{d} \frac{R_{j} S_{j}}{M_{j}}\right) .
$$

Moreover, for all $R \in G$ we have

$$
E\left(\sum_{j=1}^{d} \frac{R_{j} S_{j}}{M_{j}}\right)=1 .
$$

Consequently, $S \in H_{0}$. This proves $H=H_{0}$ and also completes the proof of Theorem 2.1.

\section{Proof of Theorem 2.2}

4.1. Preliminaries. The first lemma shows how we can extract a digit $D_{Q, j}(A)$ with help of exponential sums.

Lemma 4.1. Suppose that $Q \in \mathbb{F}_{q}[T]$ with $\operatorname{deg} Q=k \geq 1$. Set

$$
c_{H, D}=\frac{1}{q^{k}} E\left(-\frac{D H}{Q}\right) .
$$

Then

$$
\sum_{H \in P_{k}} c_{H, D} E\left(\frac{A H}{Q^{j+1}}\right)= \begin{cases}1 & \text { if } D_{Q, j}(A)=D \\ 0 & \text { if } D_{Q, j}(A) \neq D .\end{cases}
$$

Proof. Consider the $Q$-ary expansion

$$
A=\sum_{j \geq 0} D_{Q, j}(A) Q^{j} \quad \text { with } \quad D_{Q, j}(A) \in P_{k} .
$$

Then it follows that for $H \in P_{k}$

$$
E\left(\frac{A H}{Q^{j+1}}\right)=E\left(\frac{D_{Q, j}(A) H}{Q}\right) .
$$


Consequently, for every $D \in P_{k}$ we obtain

$$
\begin{aligned}
\sum_{H \in P_{k}} c_{H, D} E\left(\frac{A H}{Q^{j+1}}\right) & =\frac{1}{q^{k}} \sum_{H \in P_{k}} E\left(-\frac{D H}{Q}\right) E\left(\frac{A H}{Q^{j+1}}\right) \\
& =\frac{1}{q^{k}} \sum_{H \in P_{k}} E\left(\frac{H}{Q}\left(D_{Q, j}(A)-D\right)\right) \\
& = \begin{cases}1 & \text { if } D_{Q, j}(A)=D, \\
0 & \text { if } D_{Q, j}(A) \neq D .\end{cases}
\end{aligned}
$$

The next two lemmas are slight variations of estimates of [2].

Lemma 4.2. Suppose that $Q \in \mathbb{F}_{q}[T]$ has degree $\operatorname{deg} Q=k \geq 1$ and that $P \in \mathbb{F}_{q}[T]$ is a polynomial of degree $\operatorname{deg} P=r \geq 1$. Then

$$
\begin{aligned}
\frac{1}{q^{n}} & \left|\sum_{A \in P_{n}} E\left(\frac{H}{Q^{j+1}} P(A)\right)\right| \\
& \ll n^{2^{2-r}} \max \left(q^{-(j+1) k 2^{-r}}, q^{-n 2^{-r}}, q^{(j+1) k 2^{-r}-n r 2^{-r}}\right)
\end{aligned}
$$

Corollary 4.1. Let $n^{1 / 3} \leq j+1 \leq \frac{r n}{k}-n^{1 / 3}$. Then there exists a constant $c>0$ such that uniformly in that range

$$
\frac{1}{q^{n}}\left|\sum_{A \in P_{n}} E\left(\frac{H}{Q^{j+1}} P(A)\right)\right| \ll e^{-c n^{1 / 3}} .
$$

A similar estimate holds for monic irreducible polynomials $I_{n}$ of degree $<n$. Note that $\left|I_{n}\right|=q^{n} /((q-1) n)+O\left(q^{n / 2}\right) \sim q^{n} /((q-1) n)$.

Lemma 4.3. Let $\frac{2 r}{k} n^{1 / 3} \leq j+1 \leq \frac{r n}{k}-\frac{2 r}{k} n^{1 / 3}$, and $H$ be a polynomial coprime to $Q$. Then

$$
\frac{1}{\left|I_{n}\right|}\left|\sum_{A \in I_{n}} E\left(\frac{H}{Q^{j+1}} P(A)\right)\right| \ll(\log n) \cdot n^{4 / 3+2^{-2-2 r}} q^{-r 2^{-2 r} n^{1 / 3}}
$$

With help of theses estimates we can prove the following frequency estimates.

Lemma 4.4. Let $m$ be a fixed integer and $\frac{2 r}{k} n^{1 / 3} \leq j_{1}<j_{2}<\cdots<j_{m} \leq$ $\frac{n r}{k}-\frac{2 r}{k} n^{1 / 3}$. Then

$$
\begin{aligned}
\frac{1}{q^{n}} \cdot \#\left\{A \in P_{n}: D_{Q, j_{1}}(P(A))=D_{1}, \ldots, D_{Q, j_{m}}(P(A))=D_{m}\right\} \\
\quad=\frac{1}{q^{k m}}+O\left(e^{-c n^{1 / 3}}\right)
\end{aligned}
$$


and

$$
\begin{aligned}
\frac{1}{\left|I_{n}\right|} \cdot & \#\left\{A \in I_{n}: D_{Q, j_{1}}(P(A))=D_{1}, \ldots, D_{Q, j_{m}}(P(A))=D_{m}\right\} \\
& =\frac{1}{q^{k m}}+O\left(e^{-c n^{1 / 3}}\right)
\end{aligned}
$$

uniformly for all $D_{1}, \ldots, D_{m} \in P_{k}$ and for all $j_{1}, \ldots, j_{m}$ in the mentioned range.

Proof. By Lemma 4.1 we have

$$
\begin{aligned}
& \frac{1}{q^{n}} \#\left\{A \in P_{n}: D_{Q, j_{1}}(P(A))=D_{1}, \ldots, D_{Q, j_{m}}(P(A))=D_{m}\right\}= \\
& =\frac{1}{q^{n}} \sum_{A \in P_{n}} \prod_{i=1}^{m}\left(\sum_{H_{i} \in P_{k}} c_{H_{i}, D_{i}} E\left(\frac{H_{i}}{Q^{j_{i}+1}} P(A)\right)\right) \\
& =\sum_{H_{1}, \ldots, H_{m} \in P_{k}} \prod_{i=1}^{m} c_{H_{i}, D_{i}} \frac{1}{q^{n}} \sum_{A \in P_{n}} E\left(P(A)\left(\frac{H_{1}}{Q^{j_{1}+1}}+\cdots+\frac{H_{m}}{Q^{j_{m}+1}}\right)\right) \\
& =c_{0, D_{1}}^{\cdots} \cdots c_{0, D_{m}}{ }^{*}{ }^{m} \sum_{H_{1}, \ldots, H_{m} \in P_{k}} \prod_{i=1}^{m} c_{H_{i}, D_{i}} \frac{1}{q^{n}} \sum_{A \in P_{n}} E\left(P(A)\left(\frac{H_{1}}{Q^{j_{1}+1}}+\cdots+\frac{H_{m}}{Q^{j_{m}+1}}\right)\right) \\
& =\frac{1}{q^{k m}}+S,
\end{aligned}
$$

where $\sum^{*}$ denotes that we sum just over all $\left(H_{1}, \ldots, H_{m}\right) \neq(0, \ldots, 0)$. In order to complete the proof we just have to show that $S=O\left(e^{-c n^{1 / 3}}\right)$.

Let $l$ be the largest $i$ with $H_{i} \neq 0$ then

$$
\frac{1}{q^{n}} \sum_{A \in P_{n}} E\left(P(A)\left(\frac{H_{1}}{Q^{j_{1}+1}}+\cdots+\frac{H_{m}}{Q^{j_{m}+1}}\right)\right)=\frac{1}{q^{n}} \sum_{A \in P_{n}} E\left(P(A) \frac{H}{Q^{j_{l}+1}}\right)
$$

where $H=H_{l}+H_{l-1} Q^{j_{l}-j_{l-1}}+\cdots+H_{1} Q^{j_{l}-j_{1}}$. By our assumption we have $\frac{2 r}{k} n^{1 / 3} \leq j_{l} \leq \frac{n r}{k}-\frac{2 r}{k} n^{1 / 3}$. Hence by Lemma 4.2 the first result follows.

The proof for $A \in I_{n}$ is completely the same. 
4.2. Weak Convergence. The idea of the proof of Theorem 2.2 is to compare the distribution of $g(P(A))$ with the distribution of sums of independent identically distributed random variables. Let $Y_{0}, Y_{1}, \ldots$ be independent identically distributed random variables on $P_{k}$ with $\mathbb{P}\left[Y_{j}=D\right]=q^{-k}$ for all $D \in P_{k}$. Then Lemma 4.4 can be rewritten as

$$
\begin{gathered}
\frac{1}{q^{n}} \#\left\{A \in P_{n}: D_{Q, j_{1}}(P(A))=D_{1}, \ldots, D_{Q, j_{m}}(P(A))=D_{m}\right\} \\
=\mathbb{P}\left[Y_{j_{1}}=D_{1}, \ldots, Y_{j_{m}}=D_{m}\right]+O\left(e^{-c n^{1 / 3}}\right) .
\end{gathered}
$$

Note further that this relation is also true if $j_{1}, \ldots, j_{m}$ vary in the range $\frac{2 r}{k} n^{1 / 3} \leq j_{1}, j_{2}, \cdots, j_{m} \leq \frac{n r}{k}-\frac{2 r}{k} n^{1 / 3}$ and are not ordered. It is even true if some of them are equal.

In fact, we will use a moment method, that is, we will show that the moments of $g(P(A))$ can be compared with moments of the normal distribution. Finally this will show that the corresponding (normalized) distribution function of $g(P(A))$ converges to the normal distribution function $\Phi(x)$.

It turns out that we will have to cut off the first and last few digits, that is, we will work with

$$
\widetilde{g}(P(A)):=\sum_{\frac{2 r}{k} n^{1 / 3} \leq j \leq \frac{n r}{k}-\frac{2 r}{k} n^{1 / 3}} g\left(D_{Q, j}(P(A))\right)
$$

instead of $g(P(A))$.

Lemma 4.5. Set

$$
\mu=\frac{1}{q^{k}} \sum_{H \in P_{k}} g(H)=\mathbb{E} g\left(Y_{j}\right) .
$$

Then the m-th (central) moment of $\widetilde{g}(P(A))$ is given by

$$
\begin{gathered}
\frac{1}{q^{n}} \sum_{A \in P_{n}}\left(\widetilde{g}(P(A))-\left(\frac{n r}{k}-2 \frac{2 r}{k} n^{1 / 3}\right) \mu\right)^{m}= \\
=\mathbb{E}\left(\sum_{\frac{2 r}{k} n^{1 / 3} \leq j \leq \frac{n r}{k}-\frac{2 r}{k} n^{1 / 3}}\left(g\left(Y_{j}\right)-\mu\right)\right)^{m}+O\left(n^{m} e^{-c n^{1 / 3}}\right) .
\end{gathered}
$$


Proof. For notational convenience we just consider the second moment:

$$
\begin{aligned}
\frac{1}{q^{n}} & \sum_{A \in P_{n}}\left(\widetilde{g}(P(A))-\left(\frac{n r}{k}-\frac{2 r}{k} n^{1 / 3}\right) \mu\right)^{2}= \\
= & \sum_{j_{1}, j_{2}} \sum_{D_{1}, D_{2}} g\left(D_{1}\right) g\left(D_{2}\right) \frac{1}{q^{n}} \#\left\{A \in P_{n}: D_{Q, j_{i}}(P(A))=D_{i}, i=1,2\right\} \\
& -\sum_{j_{1}} \sum_{D_{1}} g\left(D_{1}\right) \frac{1}{q^{n}} \#\left\{A \in P_{n}: D_{Q j_{1}}(P(A))=D_{1}\right\} \cdot \sum_{j_{2}} \mu \\
& -\sum_{j_{1}} \mu \sum_{j_{2}} \sum_{D_{2}} g\left(D_{2}\right) \frac{1}{q^{n}} \#\left\{A \in P_{n}: D_{Q, j_{2}}(P(A))=D_{2}\right\}+\sum_{j_{1}, j_{2}} \mu^{2} \\
= & \sum_{j_{1}, j_{2}} \sum_{D_{1}, D_{2}} g\left(D_{1}\right) g\left(D_{2}\right) \mathbb{P}\left[Y_{j_{1}}=D_{1}, Y_{j_{2}}=D_{2}\right]+O\left(n^{2} e^{-c n^{1 / 3}}\right) \\
& -\sum_{j_{1}} \sum_{D_{1}} g\left(D_{1}\right) \mathbb{P}\left[Y_{j_{1}}=D_{1}\right] \sum_{j_{2}} \mu \\
& -\sum_{j_{1}} \mu \sum_{j_{2}} \sum_{D_{2}} g\left(D_{2}\right) \mathbb{P}\left[Y_{j_{2}}=\varepsilon_{2}\right]+\sum_{j_{1}} \sum_{j_{2}} \mu^{2} \\
= & \mathbb{E}\left(\sum_{j}\left(g\left(Y_{j}\right)-\mu\right)\right)^{2}+O\left(n^{2} e^{-c n^{1 / 3}}\right) .
\end{aligned}
$$

The very same procedure works in general and completes the proof of the lemma.

Since the sum of independent identically distributed random variables converges (after normalization) to the normal distribution it follows from Lemma 4.5

$$
\frac{1}{q^{n}} \#\left\{A \in P_{n}: \frac{\widetilde{g}(P(A))-\left(\frac{n r}{k}-\frac{2 r}{k} n^{1 / 3}\right) \mu}{\sqrt{\left(\frac{n r}{k}-\frac{2 r}{k} n^{1 / 3}\right) \sigma^{2}}} \leq x\right\}=\Phi(x)+o(1)
$$

Because of

$$
|\widetilde{g}(P(A))-g(P(A))| \ll n^{1 / 3}
$$

and $n^{1 / 3} / n^{1 / 2}=n^{-1 / 6} \rightarrow 0$ it also follows that

$$
\frac{1}{q^{n}} \#\left\{A \in P_{n}: \frac{g(P(A))-\frac{n r}{k} \mu}{\sqrt{\frac{n r}{k} \sigma^{2}}} \leq x\right\}=\Phi(x)+o(1) .
$$

This completes the proof of Theorem 2.2. 


\section{Proof of Theorem 2.3}

5.1. Preliminaries. As usual, let $\nu\left(\frac{A}{B}\right)=\operatorname{deg}(B)-\operatorname{deg}(A)$ be the valuation on $\mathbb{F}_{q}(T)$.

Lemma 5.1. For $a, b \in \mathbb{F}_{q}(T)$ we have

$$
\nu(a+b) \geq \min \{\nu(a), \nu(b)\} .
$$

Moreover, if $\nu(a) \neq \nu(b)$, then

$$
\nu(a+b)=\min \{\nu(a), \nu(b)\} .
$$

Furthermore, we will use the following easy property (see [10]) that is closely related to Lemma 3.1.

Lemma 5.2. Suppose that $\nu\left(\frac{B}{C}\right)>0$ and that $n \geq \nu\left(\frac{B}{C}\right)$, then

$$
\sum_{A \in P_{n}} E\left(\frac{B}{C} A\right)=0 .
$$

Another important tool is Mason's theorem (see [16]).

Lemma 5.3. Let $K$ be an arbitrary field and $A, B, C \in K[T]$ relatively prime polynomials with $A+B=C$. If the derivatives $A^{\prime}, B^{\prime}, C^{\prime}$ are not all zero then the degree $\operatorname{deg} C$ is smaller than the number of different zeros of $A B C$ (in a proper algebraic closure of $K$ ).

We will use Mason's theorem in order to prove the following property.

Lemma 5.4. Let $Q_{1}, Q_{2} \in \mathbb{F}_{q}[T]$ be coprime polynomials with degrees $\operatorname{deg}\left(Q_{i}\right)=k_{i} \geq 1$ such that at least one of the derivatives $Q_{1}^{\prime}, Q_{2}^{\prime}$ is nonzero. Then there exists a constant $c$ such that for all polynomials $H_{1} \in P_{k_{1}}$ and $H_{2} \in P_{k_{2}}$ with $\left(H_{1}, H_{2}\right) \neq(0,0)$ and for all integers $m_{1}, m_{2} \geq 1$ we have

$$
\operatorname{deg}\left(H_{1} Q_{2}^{m_{2}}+H_{2} Q_{1}^{m_{1}}\right) \geq \max \left\{\operatorname{deg}\left(H_{1} Q_{2}^{m_{2}}\right), \operatorname{deg}\left(H_{2} Q_{1}^{m_{1}}\right)\right\}-c .
$$

Proof. Set $A=H_{1} Q_{2}^{m_{2}}, B=H_{2} Q_{1}^{m_{1}}$, and $C=A+B$. If $A$ and $B$ are coprime by Mason's theorem we have $\operatorname{deg}(A) \leq n_{0}(A B C)-1$ and $\operatorname{deg}(B) \leq n_{0}(A B C)-1$, where $n_{0}(F)$ is defined to be the number of distinct zeroes of $F$. Hence

$$
\begin{aligned}
\max \{\operatorname{deg}(A), \operatorname{deg}(B)\} & \leq n_{0}(A B C)-1 \\
& =n_{0}\left(H_{1} H_{2} Q_{1} Q_{2} C\right)-1 \\
& \leq \operatorname{deg}\left(H_{1} H_{2} Q_{1} Q_{2}\right)+\operatorname{deg}(C)-1
\end{aligned}
$$

and consequently

$$
\operatorname{deg}(C) \geq \max \{\operatorname{deg}(A), \operatorname{deg}(B)\}-\operatorname{deg}\left(H_{1} H_{2} Q_{1} Q_{2}\right)+1 .
$$

This shows that (in the present case) $c=2 k_{1}+2 k_{2}$ is surely a proper choice. 
If $A$ and $B$ are not coprime then by assumption the common factor $D$ is surely a divisor of $H_{1} H_{2}$. Furthermore, there exists $m^{\prime} \geq 0$ such that $D^{2}$ is a divisor of $H_{1} H_{2}\left(Q_{1} Q_{2}\right)^{m^{\prime}}$. Consequently we have

$$
(A / D)(B / D)=\left(H_{1} H_{2}\left(Q_{1} Q_{2}\right)^{m^{\prime}} / D^{2}\right) Q_{1}^{m_{1}-m^{\prime}} Q_{2}^{m_{2}-m^{\prime}}
$$

and by a reasoning as above we get

$$
\begin{aligned}
& \operatorname{deg}(C / D) \geq \\
& \quad \max \{\operatorname{deg}(A / D), \operatorname{deg}(B / D)\}-\operatorname{deg}\left(\left(H_{1} H_{2}\left(Q_{1} Q_{2}\right)^{m^{\prime}} / D^{2}\right) Q_{1} Q_{2}\right)+1 .
\end{aligned}
$$

or

$$
\operatorname{deg}(C) \geq \max \{\operatorname{deg}(A), \operatorname{deg}(B)\}-\operatorname{deg}\left(\left(H_{1} H_{2}\left(Q_{1} Q_{2}\right)^{m^{\prime}} / D^{2}\right) Q_{1} Q_{2}\right)+1
$$

Since there are only finitely possibilities for $H_{1}, H_{2}$, and $D$ the lemma follows.

5.2. Convergence of Moments. The idea of the proof of Theorem 2.3 is completely the same as that of Theorem 2.2. We prove weak convergence by considering moments. The first step is to provide a generalization of Lemma 4.4.

Lemma 5.5. Let $m_{1}, m_{2}$ be fixed integers. Then there exists a constant $c^{\prime}>0$ such that for all $0 \leq i_{1}<i_{2}<\cdots<i_{m_{1}} \leq \frac{n}{k_{1}}-c^{\prime}$ and $0 \leq j_{1}<j_{2}<$ $\cdots<j_{m_{2}} \leq \frac{n}{k_{2}}-c^{\prime}$ we have

$$
\begin{aligned}
& \frac{1}{q^{n}} \#\left\{A \in P_{n}: D_{Q_{1}, i_{1}}(A)=D_{1}, \ldots, D_{Q_{1}, i_{m_{1}}}(A)=D_{m_{1}},\right. \\
& \left.D_{Q_{2}, j_{1}}(A)=E_{1}, \ldots, D_{Q_{2}, j_{m_{2}}}(A)=E_{m_{2}}\right\} \\
& =\frac{1}{q_{1}^{k_{1} m_{1}} q_{2}^{k_{2} m_{2}}} .
\end{aligned}
$$

Instead of giving a complete proof of this lemma we will concentrate on the cases $m_{1}=m_{2}=1$ and $m_{1}=m_{2}=2$. The general case runs along the same lines (but the notation will be terrible). 
First let $m_{1}=m_{2}=1$. Here we have

$$
\begin{aligned}
\frac{1}{q^{n}} \#\left\{A \in P_{n}: D_{Q_{1}, i}(A)=D_{1}, D_{Q_{2}, j}(A)=E_{1}\right\} \\
=\frac{1}{q^{n}} \sum_{A \in P_{n}} \sum_{H_{1} \in P_{k_{1}}} c_{H_{1}, Q_{1}, D_{1}} E\left(\frac{A H_{1}}{Q_{1}^{i+1}}\right) \sum_{H_{2} \in P_{k_{2}}} c_{H_{2}, Q_{2}, E_{1}} E\left(\frac{A H_{2}}{Q_{2}^{j+1}}\right) \\
=\frac{1}{q^{k_{1}+k_{2}}} \\
\quad+\sum_{\left(H_{1}, H_{2}\right) \neq(0,0)} c_{H_{1}, Q_{1}, D_{1}} c_{H_{2}, Q_{2}, E_{1}} \frac{1}{q^{n}} \sum_{A \in P_{n}} E\left(A\left(\frac{H_{1}}{Q_{1}^{i+1}}+\frac{H_{2}}{Q_{2}^{j+1}}\right)\right) .
\end{aligned}
$$

Now we can apply Lemma 5.4 and obtain

$$
\begin{aligned}
\nu\left(\frac{H_{1}}{Q_{1}^{i+1}}+\frac{H_{2}}{Q_{2}^{j+1}}\right)= & \nu\left(\frac{H_{1} Q_{2}^{j+1}+H_{2} Q_{1}^{i+1}}{Q_{1}^{i+1} Q_{2}^{j+1}}\right) \\
\leq & k_{1}(i+1)+k_{2}(j+1) \\
& -\max \left\{\operatorname{deg}\left(H_{1}\right)+k_{2}(j+1), \operatorname{deg}\left(H_{1}\right)+k_{1}(i+1)\right\} \\
& +c \\
\leq & \min \left\{k_{1}(i+1), k_{2}(j+1)\right\}+c .
\end{aligned}
$$

Thus, there exists a constant $c^{\prime}>0$ such that

$$
\min \left\{k_{1}(i+1), k_{2}(j+1)\right\}+c \leq n
$$

for all $i, j$ with $0 \leq i \leq \frac{n}{k_{1}}-c^{\prime}$ and $0 \leq j \leq \frac{n}{k_{2}}-c^{\prime}$. Hence, by Lemma 5.2

$$
\sum_{A \in P_{n}} E\left(A\left(\frac{H_{1}}{Q_{1}^{i+1}}+\frac{H_{2}}{Q_{2}^{j+1}}\right)\right)=0
$$

This completes the proof for the case $m_{1}=m_{2}=1$. 
Next suppose that $m_{1}=m_{2}=2$. Here we have

$$
\begin{aligned}
& \frac{1}{q^{n}} \#\left\{A \in P_{n}: D_{Q_{1}, i_{1}}(A)=D_{1}, D_{Q_{1}, i_{2}}(A)=D_{2},\right. \\
& \left.D_{Q_{2}, j_{1}}(A)=E_{1}, D_{Q_{2}, j_{2}}(A)=E_{2}\right\} \\
& =\frac{1}{q^{n}} \sum_{A \in P_{n}}\left(\sum_{H_{11} \in P_{k_{1}}} c_{Q_{1}, H_{11}, D_{1}} E\left(\frac{H_{11}}{Q_{1}^{i_{1}+1}} A\right)\right) \times \\
& \left(\sum_{H_{12} \in P_{k_{1}}} c_{Q_{1}, H_{12}, D_{2}} E\left(\frac{H_{12}}{Q_{1}^{i_{2}+1}} A\right)\right) \times \\
& \left(\sum_{H_{21} \in P_{k_{2}}} c_{Q_{2}, H_{21}, E_{1}} E\left(\frac{H_{21}}{Q_{2}^{j_{1}+1}} A\right)\right) \times \\
& \left(\sum_{H_{22} \in P_{k_{2}}} c_{Q_{2}, H_{22}, E_{2}} E\left(\frac{H_{22}}{Q_{2}^{j_{2}+1}} A\right)\right) \\
& =\sum_{H_{11}, H_{12} \in P_{k_{1}}, H_{21}, H_{22} \in P_{k_{2}}} c_{Q_{1}, H_{11}, D_{1}} c_{Q_{1}, H_{12}, D_{2}} c_{Q_{2}, H_{21}, E_{1}} c_{Q_{2}, H_{22}, E_{2}} \\
& \times \frac{1}{q^{n}} \sum_{A \in P_{n}} E\left(A\left(\frac{H_{11}}{Q_{1}^{i_{1}+1}}+\frac{H_{12}}{Q_{1}^{i_{2}+1}}+\frac{H_{21}}{Q_{2}^{j_{1}+1}}+\frac{H_{22}}{Q_{2}^{j_{2}+1}}\right)\right)
\end{aligned}
$$

Of course, if $H_{11}=H_{12}=H_{21}=H_{22}=0$ then we obtain the main term $q_{1}^{-2 k_{1}} q_{2}^{-2 k_{2}}$.

For the remaining cases we will distinguish between four cases. Note that we only consider the case where all polynomials $H_{11}, H_{12}, H_{21}, H_{22}$ are non-zero. If some (but not all) of them are zero the considerations are still easier.

Case 1. $i_{2}-i_{1} \leq c_{1}, j_{2}-j_{1} \leq c_{2}$ for properly chosen constants $c_{1}, c_{2}>0$. In this case we proceed as in the case $m_{1}=m_{2}=1$ and obtain

$$
\begin{aligned}
\nu\left(\frac{H_{11}}{Q_{1}^{i_{1}+1}}\right. & \left.+\frac{H_{12}}{Q_{1}^{i_{2}+1}}+\frac{H_{21}}{Q_{2}^{j_{1}+1}}+\frac{H_{22}}{Q_{2}^{j_{2}+1}}\right) \\
& =\nu\left(\frac{\left(H_{11} Q_{1}^{i_{2}-i_{1}}+H_{12}\right) Q_{2}^{j_{2}+1}+\left(H_{21} Q_{2}^{j_{2}-j_{1}}+H_{22}\right) Q_{1}^{i_{2}+1}}{Q_{1}^{i_{2}+1} Q_{2}^{j_{2}+1}}\right)
\end{aligned}
$$




$$
\begin{aligned}
& \leq k_{1}\left(i_{2}+1\right)+k_{2}\left(j_{2}+1\right) \\
& \quad-\max \left\{\operatorname{deg}\left(H_{11} Q_{1}^{i_{2}-i_{1}}+H_{12}\right)+k_{2}\left(j_{2}+1\right),\right. \\
& \left.\quad \operatorname{deg}\left(H_{21} Q_{2}^{j_{2}-j_{1}}+H_{22}\right)+k_{1}\left(i_{2}+1\right)\right\}+c\left(c_{1}, c_{2}\right) \\
& \leq \min \left\{k_{1}\left(i_{1}+1\right), k_{2}\left(j_{1}+1\right)\right\}+\tilde{c}\left(c_{1}, c_{2}\right)
\end{aligned}
$$

for some suitable constants $c\left(c_{1}, c_{2}\right)$ and $\tilde{c}\left(c_{1}, c_{2}\right)$.

Case 2. $i_{2}-i_{1}>c_{1}, j_{2}-j_{1}>c_{2}$ for properly chosen constants $c_{1}, c_{2}>0$

First we recall that

$$
\nu\left(\frac{H_{11}}{Q_{1}^{i_{1}+1}}+\frac{H_{21}}{Q_{2}^{j_{1}+1}}\right) \leq \min \left\{k_{1}\left(i_{1}+1\right), k_{2}\left(j_{1}+1\right)\right\}+c .
$$

Furthermore

$$
\begin{aligned}
\nu\left(\frac{H_{12}}{Q_{1}^{i_{2}+1}}\right) & \geq k_{1}\left(i_{2}+1\right)-\operatorname{deg} H_{12} \\
& \geq k_{1}\left(i_{2}-i_{1}\right)+k_{1} i_{1}>k_{1}\left(i_{1}+c_{1}\right) \\
\nu\left(\frac{H_{22}}{Q_{2}^{j_{2}+1}}\right) & >k_{2}\left(j_{1}+c_{2}\right)
\end{aligned}
$$

Thus, if $c_{1}$ and $c_{2}$ are chosen that $\left(c_{1}-1\right) k_{1}>c$ and $\left(c_{2}-1\right) k_{2}>c$ then

$$
\nu\left(\frac{H_{11}}{Q_{1}^{i_{1}+1}}+\frac{H_{21}}{Q_{2}^{j_{1}+1}}\right)<\min \left\{\nu\left(\frac{H_{12}}{Q_{1}^{i_{2}+1}}\right), \nu\left(\frac{H_{22}}{Q_{2}^{j_{2}+1}}\right)\right\}
$$

and consequently by Lemma 5.1

$$
\begin{aligned}
\nu\left(\frac{H_{11}}{Q_{1}^{i_{1}+1}}+\frac{H_{12}}{Q_{1}^{i_{2}+1}}+\frac{H_{21}}{Q_{2}^{j_{1}+1}}+\frac{H_{22}}{Q_{2}^{j_{2}+1}}\right) & =\nu\left(\frac{H_{11}}{Q_{1}^{i_{1}+1}}+\frac{H_{21}}{Q_{2}^{j_{1}+1}}\right) \\
& \leq \min \left(k_{1}\left(i_{1}+1\right), k_{2}\left(j_{1}+1\right)\right)+c
\end{aligned}
$$

Case 3. $i_{2}-i_{1} \leq c_{1}, j_{2}-j_{1}>c_{2}$ for properly chosen constants $c_{1}, c_{2}>0$

First we have

$$
\begin{aligned}
\nu\left(\frac{H_{11}}{Q_{1}^{i_{1}+1}}+\right. & \left.\frac{H_{12}}{Q_{1}^{i_{2}+1}}+\frac{H_{21}}{Q_{2}^{j_{1}+1}}\right) \\
= & \nu\left(\frac{\left(H_{11} Q_{1}^{i_{2}-i_{1}}+H_{12}\right) Q_{2}^{j_{1}+1}+H_{21} Q_{1}^{i_{2}+1}}{Q_{1}^{i_{2}+1} Q_{2}^{j_{1}+1}}\right) \\
\leq & k_{1}\left(i_{2}+1\right)+k_{2}\left(j_{1}+1\right) \\
& -\max \left\{k_{1}\left(i_{2}-i_{1}\right)+k_{2}\left(j_{1}+1\right), k_{1}\left(i_{2}+1\right)\right\}+c\left(c_{1}\right) \\
= & \min \left\{k_{1}\left(i_{1}+1\right), k_{2}\left(j_{1}+1\right)\right\}+c\left(c_{1}\right) .
\end{aligned}
$$


Furthermore,

$$
\begin{aligned}
\nu\left(\frac{H_{22}}{Q_{2}^{j_{2}+1}}\right) & \geq k_{2}\left(j_{2}+1\right)-\operatorname{deg}\left(H_{22}\right) \\
& \geq k_{2}\left(j_{2}-j_{1}\right)+k_{2} j_{1}>k_{2}\left(j_{1}+c_{2}\right) .
\end{aligned}
$$

Hence, if $c_{2}$ is sufficiently large then

$$
\begin{aligned}
\nu\left(\frac{H_{11}}{Q_{1}^{i_{1}+1}}+\frac{H_{12}}{Q_{1}^{i_{2}+1}}+\frac{H_{21}}{Q_{2}^{j_{1}+1}}+\frac{H_{22}}{Q_{2}^{j_{2}+1}}\right)= & \nu\left(\frac{H_{11}}{Q_{1}^{i_{1}+1}}+\frac{H_{12}}{Q_{1}^{i_{2}+1}}+\frac{H_{21}}{Q_{2}^{j_{1}+1}}\right) \\
< & \min \left(k_{1}\left(i_{1}+1\right), k_{2}\left(j_{1}+1\right)\right) \\
& +c\left(c_{1}\right)
\end{aligned}
$$

Case 4. $i_{2}-i_{1}>c_{1}, j_{2}-j_{1} \leq c_{2}$ for properly chosen constants $c_{1}, c_{2}>0$ This case is completely symmetric to case 3 .

Putting these four cases together they show that (with suitably chosen constants $c_{1}, c_{2}$ ) there exists a constant $\tilde{c}$ such that for all polynomials $\left(H_{11}, H_{12}, H_{21}, H_{22}\right) \neq(0,0,0,0)$ we have

$$
\nu\left(\frac{H_{11}}{Q_{1}^{i_{1}+1}}+\frac{H_{12}}{Q_{1}^{i_{2}+1}}+\frac{H_{21}}{Q_{2}^{j_{1}+1}}+\frac{H_{22}}{Q_{2}^{j_{2}+1}}\right) \leq \min \left(k_{1}\left(i_{1}+1\right), k_{2}\left(j_{1}+1\right)\right)+\tilde{c} .
$$

Thus, there exists a constant $c^{\prime}>0$ such that

$$
\min \left\{k_{1}\left(i_{1}+1\right), k_{2}\left(j_{1}+1\right)\right\}+\tilde{c} \leq n
$$

for all $i_{1}, j_{1}$ with $0 \leq i_{1} \leq \frac{n}{k_{1}}-c^{\prime}$ and $0 \leq j_{1} \leq \frac{n}{k_{2}}-c^{\prime}$. Hence, by Lemma 5.2

$$
\sum_{A \in P_{n}} E\left(A\left(\frac{H_{11}}{Q_{1}^{i_{1}+1}}+\frac{H_{12}}{Q_{1}^{i_{2}+1}}+\frac{H_{21}}{Q_{2}^{j_{1}+1}}+\frac{H_{22}}{Q_{2}^{j_{2}+1}}\right)\right)=0 .
$$

This completes the proof for the case $m_{1}=m_{2}=2$.

As in the proof of Theorem 2.2 we can rewrite Lemma 5.5 as

$$
\begin{gathered}
\frac{1}{q^{n}} \#\left\{A \in P_{n}: D_{Q_{1}, i_{1}}(A)=D_{1}, \ldots, D_{Q_{1}, i_{m_{1}}}(A)=D_{m_{1}},\right. \\
\left.D_{Q_{2}, j_{1}}(A)=E_{1}, \ldots, D_{Q_{2}, j_{m_{2}}}(A)=E_{m_{2}}\right\} \\
=\mathbb{P}\left[Y_{i_{1}}=D_{1}, \ldots, Y_{i_{m_{1}}}=D_{m_{1}}, Z_{j_{1}}=E_{1}, \ldots, Z_{j_{m_{2}}}=E_{m_{2}}\right],
\end{gathered}
$$

where $Y_{i}$ and $Z_{j}$ are independent random variables that are uniformly distributed on $P_{k_{1}}$ resp. on $P_{k_{2}}$. 
If we define

$$
\begin{aligned}
\widetilde{g_{1}}(A) & :=\sum_{j_{1} \leq \frac{n}{k_{1}}-c^{\prime}} g_{1}\left(D_{Q_{1}, j_{1}}(A)\right), \\
\widetilde{g_{2}}(A) & :=\sum_{j_{2} \leq \frac{n}{k_{2}}-c^{\prime}} g_{2}\left(D_{Q_{2}, j_{2}}(A)\right)
\end{aligned}
$$

then Lemma 5.5 immediately translates to

Lemma 5.6. For all positive integers $m_{1}, m_{2}$ we have for sufficiently large $n$

$$
\begin{aligned}
& \frac{1}{q^{n}} \sum_{A \in P_{n}}\left(\widetilde{g_{1}}(A)-\frac{n}{k_{1}} \mu_{g_{1}}\right)^{m_{1}}\left(\widetilde{g_{2}}(A)-\frac{n}{k_{2}} \mu_{g_{2}}\right)^{m_{2}} \\
& \quad=\mathbb{E}\left(\sum_{j_{1} \leq \frac{n}{k_{1}}-c^{\prime}}\left(g_{1}\left(Y_{j_{1}}\right)-\mu_{g_{1}}\right)\right)^{m_{1}} \mathbb{E}\left(\sum_{j_{2} \leq \frac{n}{k_{2}}-c^{\prime}}\left(g_{2}\left(Z_{j_{2}}\right)-\mu_{g_{2}}\right)\right)^{m_{2}} .
\end{aligned}
$$

Of course this implies that the joint distribution of $\tilde{g}_{1}$ and $\tilde{g}_{2}$ is asymptotically Gaussian (after normalization). Since the differences $g_{1}(A)-\tilde{g}_{1}(A)$ and $g_{2}(A)-\tilde{g}_{2}(A)$ are bounded the same is true for the joint distribution of $g_{1}$ and $g_{2}$. This completes the proof of Theorem 2.3.

\section{References}

[1] N. L. BAssily, I. KÁtAI, Distribution of the values of q-additive functions on polynomial sequences. Acta Math. Hung. 68 (1995), 353-361.

[2] Mireille CaR, Sommes de puissances et d'irréductibles dans $F_{q}[X]$. Acta Arith. 44 (1984), $7-34$.

[3] J. Coquet, Corrélation de suites arithmétiques. Sémin. Delange-Pisot-Poitou, 20e Année 1978/79, Exp. 15, 12 p. (1980).

[4] H. Delange Sur les fonctions q-additives ou q-multiplicatives. Acta Arith. 21 (1972), 285298.

[5] H. Delange Sur la fonction sommatoire de la fonction "Somme de Chiffres". L'Enseignement math. 21 (1975), 31-77.

[6] M. Drmota, The joint distribution of q-additive functions. Acta Arith. 100 (2001), 17-39.

[7] M. Drmota, J. Gajdosik, The distribution of the sum-of-digits function. J. Theor. Nombres Bordx. 10 (1998), 17-32.

[8] J. M. Dumont, A. Thomas, Gaussian asymptotic properties of the sum-of-digits functions. J. Number Th. 62 (1997), 19-38.

[9] P. J. Grabner, P. Kirschenhofer, H. Prodinger, R. F. Tichy, On the moments of the sum-of-digits function. in: Applications of Fibonacci Numbers 5 (1993), 263-271

[10] G.W. Effinger, D. R. HAYes, Additive number theory of polynomials over a finite field. Oxford University Press, New York, 1991.

[11] I. KÁtAI, Distribution of q-additive function. Probability theory and applications, Essays to the Mem. of J. Mogyorodi, Math. Appl. 80 (1992), Kluwer, Dortrecht, 309-318.

[12] R. E. Kennedy, C. N. Cooper, An extension of a theorem by Cheo and Yien concerning digital sums. Fibonacci Q. 29 (1991), 145-149.

[13] D.-H. KIM, On the joint distribution of q-additive functions in residue classes. J. Number Theory 74 (1999), $307-336$. 
[14] P. Kirschenhofer, On the variance of the sum of digits function. Lecture Notes Math. 1452 (1990), 112-116.

[15] E. ManstaviČIUs, Probabilistic theory of additive functions related to systems of numerations. Analytic and Probabilistic Methods in Number Theory, VSP, Utrecht 1997, 413-430.

[16] R. C. Mason, Diophantine Equations over Function Fields. London Math. Soc. Lecture Notes 96, Cambridge University Press, 1984.

[17] W. SteIner, On the joint distribution of q-additive functions on polynomial sequences, Theory of Stochastic Processes 8 (24) (2002), 336-357.

Michael Drmota

Inst. of Discrete Math. and Geometry

TU Wien

Wiedner Hauptstr. 8-10

A-1040 Wien, Austria

E-mail : michael.drmota@tuwien.ac.at

URL: http://www.dmg.tuwien.ac.at/drmota/

Georg Gutenbrunner

Inst. of Discrete Math. and Geometry

TU Wien

Wiedner Hauptstr. 8-10

A-1040 Wien, Austria

E-mail : georg@gutenbrunner.com 